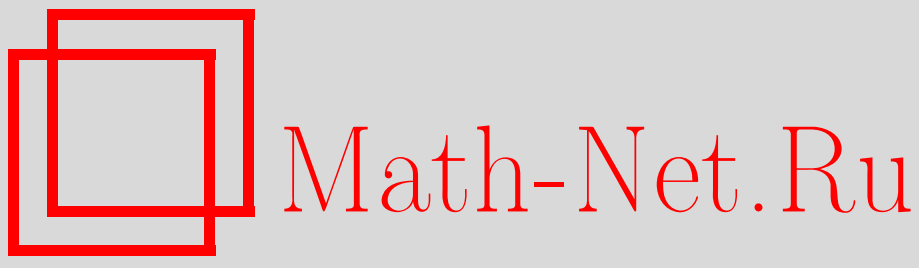

С. В. Гонченко, Модули $\Omega$-сопряженности двумерных диффеоморфизмов с негрубым гетероклиническим контуром, Матем. сб., 1996, том 187, номер 9, 3-24

DOI: https://doi.org/10.4213/sm155

Использование Общероссийского математического портала Math-Net.Ru подразумевает, что вы прочитали и согласны с пользовательским соглашением http://www . mathnet.ru/rus/agreement

Параметры загрузки:

IP: 54.80 .97 .219

26 апреля 2023 г., $12: 38: 41$ 
УДК 517.9

\author{
C.В. Гонченко
}

\title{
Модули $\Omega$-сопряженности двумерных диффеоморфизмов с негрубым гетероклиническим контуром
}

\begin{abstract}
В статье рассмотрены двумерные диффеоморфизмы, имеющие негрубый гетероклинический контур, составленный из двух седловых неподвижных точек и двух гетероклинических траекторий: грубой и негрубой. Такие диффеоморфизмы делятся на три класса, в зависимости от структуры множества $N$ траекторий, целиком лежащих в окрестности контура. В случае диффеоморфизмов первого и второго классов $N$ допускает полное описание. Показано, что диффеоморфизмы третьего класса имеют $\Omega$-модули - непрерьвные инварианты топологической сопряженности на множестве неблуждающих траекторий. Указаны явно два таких модуля: $\theta$ и $\tau_{0}$, рассмотрены достаточные условия $\Omega$-сопряженности при рациональном $\theta$, а также доказано, что на бифуркационной поверхности диффеоморфизмов третьего класса плотны системы со счетньм множеством $\Omega$-модулей.

Библиографияя: 18 названий.
\end{abstract}

\section{Введение}

Одним из важных результатов, полученных в качественной теории дифференциальных уравнений за последнее время, является открытие у негрубых многомерных систем модулей - локально непостоянных непрерьвных функционалов, значениями которых системы разбиваются на континуум классов топологической или $\Omega$-эквивалентности (топологической эквивалентности на множестве неблуждающих траекторий). Точное определение модуля см. в 33 .

Модули топологической эквивалентности в системах с простой динамикой открыл Пэлис [1]. Он обнаружил, что такими модулями обладают уже двумерные диффеоморфизмы с негрубой гетероклинической траекторией (т.е. такие диффеоморфизмы, у которых инвариантные многообразия двух разных седловых неподвижных точек имеют одностороннее касание). Системы с негрубой гомоклинической траекторией (т.е. касаются инвариантные многообразия одной и той же седловой неподвижной точки) имеют также аналогичный модуль. Но ситуация здесь оказывается более сложной. Как показано в [2]-[4], у определенного класса систем с негрубой гомоклинической траекторией существуют модули $\Omega$-эквивалентности, и более того, на бифуркационных поверхностях таких систем плотны системы со счетным множеством $\Omega$-модулей [5], [6]. Отметим, что изменение значения $\Omega$-модуля ведет к бифуркациям неблуждающих траекторий (в частности,

Работа выполнена при финансовой поддержке Российского фонда фундаментальных исследований (грант № 93-011-1787), а также Международного научного фонда (грант № 98300) 
периодических и гомоклинических). Сушествование же счетного множества таких модулей свидетельствует, что независимое изменение их значений (как управляющих параметров) может приводить к сколь угодно вырожденным бифуркациЯM $[5],[6]$.

В настоящей статье рассматривается вопрос о сушествовании $\Omega$-модулей у двумерных диффеоморфизмов с негрубым гетероклиническим контуром, т.е. таких диффеоморфизмов, которые имеют две грубые седловые неподвижные точки, одна пара инвариантных многообразий которых имеет квадратичное касание, а другая - трансверсальное пересечение. Аналогично случаю систем с негрубой гомоклинической траекторией [7], данные диффеоморфизмы разбиваются на три класса в зависимости от структуры множества $N$ траекторий, целиком лежащих в малой окрестности гетероклинического контура. При этом показывается, что $N$ описывается полностью в случае диффеоморфизмов первого и второго классов. Но, если для диффеоморфизмов первого класса множество $N$ имеет тривиальную структуру (теорема 2), то в случае диффеоморфизмов второго класса $N$ сопряжено некоторой нетривиальной фактор-системе топологической схемы Бернулли из четырех символов (теорема 3 ). В случае диффеоморфизмов третьего класса в множестве $N$ содержатся нетривиальные гиперболические подмножества (теоремы 1 и 4), но $N$, как правило, не допускает полного описания, и более того, его структура "непрерывно" меняется при переходе от одной системы третьего класса к другой. Основная причина этого - сушествование у диффеоморфизмов третьего класса модулей $\Omega$-сопряженности. В статье явно указаны два основных $\Omega$-модуля $\theta$ и $\tau_{0}$ (теоремы 5 и 6). Кроме того, установлены некоторые достаточные условия $\Omega$-сопряженности при рациональном $\theta$ (теоремы 7 и 8), а также доказано, что на бифуркационной поверхности диффеоморфизмов третьего класса плотны системы со счетньм множеством $\Omega$-модулей (теорема 9 ).

\section{§1. Постановка задачи и некоторые технические результаты}

Рассмотрим $C^{r}$-гладкий $(r \geqslant 3)$ двумерный диффеоморфизм $f$, имеюший две грубые седловые неподвижные точки $O_{1}$ и $O_{2}$ с собственными значениями $\lambda_{s}, \gamma_{s}$ такими, что $\left|\lambda_{s}\right|<1<\left|\gamma_{s}\right|, s=1,2$. Будем предполагать, что $W^{u}\left(O_{1}\right)$ пересекается трансверсально с $W^{s}\left(O_{2}\right)$ по точкам некоторой гетероклинической траектории $\Gamma_{12}$, а $W^{u}\left(O_{2}\right)$ имеет квадратичное касание с $W^{s}\left(O_{1}\right)$ в точках некоторой гетероклинической траектории $\Gamma_{21}$ (рис. 1). В этой ситуации будем говорить, что $f$ имеет негрубый гетероклинический контур $C=O_{1} \cup O_{2} \cup \Gamma_{12} \cup \Gamma_{21}$.

Рассмотрим достаточно малую окрестность $U$ контура $C$. Она представляет собой объединение двух малых дисков $U_{1}$ и $U_{2}$, содержащих точки $O_{1}$ и $O_{2}$, и некоторого конечного числа малых окрестностей находящихся вне $U_{1}$ и $U_{2}$ точек траекторий $\Gamma_{12}$ и $\Gamma_{21}$.

1.1. Отображения в окрестности седловых неподвижных точек. В [3], [4] показано, что на $U_{s}$ существуют такие локальные $C^{r-1}$-координаты $\left(x_{s}, y_{s}\right)$, что отображения $\left.T_{0 s} \equiv f\right|_{U_{s}}, s=1,2$, имеют вид

$$
\begin{aligned}
& \bar{x}_{s}=\lambda_{s} x_{s}+f_{s}\left(x_{s}, y_{s}\right) x_{s} y_{s} \\
& \bar{y}_{s}=\gamma_{s} y_{s}+g_{s}\left(x_{s}, y_{s}\right) x_{s} y_{s}
\end{aligned}
$$




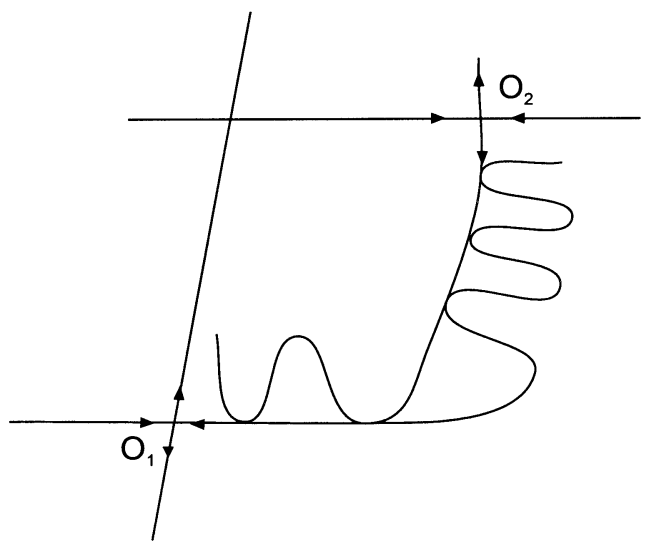

Рис. 1

где $f_{s}\left(0, y_{s}\right) \equiv 0, g_{s}\left(x_{s}, 0\right) \equiv 0$. Таким образом, уравнения многообразий $W_{\mathrm{loc}}^{s}\left(O_{s}\right)$ и $W_{\mathrm{loc}}^{u}\left(O_{s}\right)$ в данных координатах будут, соответственно, $y_{s}=0$ и $x_{s}=0$. Вид (1.1) удобен также тем, что в данных координатах отображение $T_{0 s}^{k}$ при больших $k$ будет линейным в главном порядке. Именно, имеет место следуюшее представление [3], [4] отображения $T_{0 s}^{k}:\left(x_{s}, y_{s}\right) \rightarrow\left(\bar{x}_{s}, \bar{y}_{s}\right)$ :

$$
\begin{aligned}
& \bar{x}_{s}=\lambda_{s}^{k}\left(x_{s}+\left(\widetilde{\lambda}_{s}^{k}+\widetilde{\gamma}_{s}^{-k}\right) \xi_{k s}\left(x_{s}, \bar{y}_{s}\right)\right), \\
& y_{s}=\gamma_{s}^{-k}\left(\bar{y}_{s}+\left(\widetilde{\lambda}_{s}^{k}+\widetilde{\gamma}_{s}^{-k}\right) \eta_{k s}\left(x_{s}, \bar{y}_{s}\right)\right),
\end{aligned}
$$

где $\widetilde{\lambda}_{s}=\left|\lambda_{s}\right|, \widetilde{\gamma}_{s}=\left|\gamma_{s}\right|$, если $\left|\lambda_{s} \gamma_{s}\right| \neq 1$, или $\widetilde{\lambda}_{s}=\left|\lambda_{s}\right|+\varepsilon_{k}, \widetilde{\gamma}_{s}=\left|\gamma_{s}\right|-\varepsilon_{k}$ и $\varepsilon_{k} \rightarrow 0$ при $k \rightarrow \infty$, если $\left|\lambda_{s} \gamma_{s}\right|=1$. Функции $\xi_{k s}$ и $\eta_{k s}$ равномерно ограничены по $k$ вместе с производными до порядка $(r-2)$, а производные порядка $(r-1)$ от правых частей в (1.2) стремятся к нулю при $k \rightarrow \infty$.

Выберем в $U_{1}$ пару точек $M_{1}^{+}\left(x_{1}^{+}, 0\right)$ и $M_{1}^{-}\left(0, y_{1}^{-}\right)$, принадлежащих траекториям $\Gamma_{21}$ и $\Gamma_{12}$, соответственно. В $U_{2}$ также рассмотрим пару точек $M_{2}^{+}\left(x_{2}^{+}, 0\right)$ и $M_{2}^{-}\left(0, y_{2}^{-}\right)$, принадлежаших траекториям $\Gamma_{12}$ и $\Gamma_{21}$, соответственно. Пусть $\Pi_{s}^{+} \subset U_{s}$ и $\Pi_{s}^{-} \subset U_{s}-$ достаточно малые прямоугольные окрестности точек $M_{s}^{+}$ и $M_{s}^{-}$. Координаты на $\Pi_{s}^{+}$будем обозначать как $\left(x_{0 s}, y_{0 s}\right)$, а координаты на $\Pi_{s}^{-}$ - как $\left(x_{1 s}, y_{1 s}\right)$.

Заметим, что все траектории множества $N$, за исключением $O_{1}$ и $O_{2}$, должны пересекать окрестности $\Pi_{s}^{+}$и $\Pi_{s}^{-}$(иначе эти траектории не будут близки к траекториям контура $C$ ). Однако, не для каждой точки на $\Pi_{s}^{+}$какая-либо ее итерация относительно отображения $f$ попадет в $\Pi_{s}^{-}$. Множество начальных точек на $\Pi_{s}^{+}$, траектории которых попадают в $\Pi_{s}^{-}$, как это легко видно из $(1.2)$, состоит из счетного множества полосок $\sigma_{k}^{0 s}=\Pi_{s}^{+} \cap T_{0 s}^{-k} \Pi_{s}^{-}, k=\bar{k}_{s}, \bar{k}_{s}+1, \ldots$, накапливающихся к $W_{\mathrm{loc}}^{s}\left(O_{s}\right)$. Соответственно, образы полосок $\sigma_{k}^{0 s}$ относительно отображений $T_{0 s}^{k}$ - это полоски $\sigma_{k}^{1 s} \equiv T_{0 s}^{k}\left(\sigma_{k}^{0 s}\right)$ на $\Pi_{s}^{-}$, которые накапливаются к $W_{\mathrm{loc}}^{u}\left(O_{s}\right)$.

1.2. Отображения в окрестности гетероклинических орбит. Очевидно, существуют такие натуральные числа $n_{1}$ и $n_{2}$, что $f^{n_{1}}\left(M_{1}^{-}\right)=M_{2}^{+}, f^{n_{2}}\left(M_{2}^{-}\right)=M_{1}^{+}$. 
Рассмотрим отображения $T_{12} \equiv f^{n_{1}}: \Pi_{1}^{-} \rightarrow U_{2}$ и $T_{21} \equiv f^{n_{2}}: \Pi_{2}^{-} \rightarrow U_{1}$ по траекториям, близким к $\Gamma_{12}$ и $\Gamma_{21}$, соответственно.

Поскольку $T_{12}\left(M_{1}^{-}\right)=M_{2}^{+}$, то $T_{12}$ может быть представлено в виде

$$
\begin{gathered}
\bar{x}_{02}-x_{2}^{+}=F_{12}\left(x_{11}, y_{11}-y_{1}^{-}\right) \equiv a_{12} x_{11}+b_{12}\left(y_{11}-y_{1}^{-}\right)+\cdots \\
\bar{y}_{02}=G_{12}\left(x_{11}, y_{11}-y_{1}^{-}\right) \equiv c_{12} x_{11}+d_{12}\left(y_{11}-y_{1}^{-}\right)+\cdots
\end{gathered}
$$

где $F_{12}(0,0)=0, G_{12}(0,0)=0$. Поскольку отображение $T_{12}-$ диффеоморфизм, то его якобиан, вычисленный в точке $M_{1}^{-}$, отличен от нуля, т.е. $a_{12} d_{12}-b_{12} c_{12} \neq 0$. Так как $W^{u}\left(O_{1}\right)$ пересекает $W^{s}\left(O_{2}\right)$ в точке $M_{2}^{+}$трансверсально, то $d_{12} \neq 0$.

Поскольку $T_{21}\left(M_{2}^{-}\right)=M_{1}^{+}$, то $T_{21}$ можно записать в виде

$$
\begin{gathered}
\bar{x}_{01}-x_{1}^{+}=F_{21}\left(x_{12}, y_{12}-y_{2}^{-}\right) \equiv a_{21} x_{12}+b_{21}\left(y_{12}-y_{2}^{-}\right)+\cdots, \\
\bar{y}_{01}=G_{21}\left(x_{12}, y_{12}-y_{2}^{-}\right) \equiv c_{21} x_{12}+d_{21}\left(y_{12}-y_{2}^{-}\right)^{2}+\cdots
\end{gathered}
$$

где $F_{21}(0,0)=0, G_{21}(0,0)=\partial G_{21}(0,0) / \partial y_{12}=0$. Поскольку касание $W^{u}\left(O_{2}\right)$ и $W^{s}\left(O_{1}\right)$ в точке $M_{1}^{+}$квадратичное, то $d_{21} \neq 0$. Кроме того, так как $T_{21}$ диффеоморфизм, то $b_{21} c_{21} \neq 0$.

1.3. Специальная окрестность гетероклинического контура. В качестве окрестности гетероклинического контура удобно выбрать специальную окрестность (по аналогии со специальной окрестностью негрубой гомоклинической траектории [8], [9]). Именно, выберем достаточно большие целые $\bar{k}_{1}$ и $\bar{k}_{2}$ и ограничимся рассмотрением только тех траекторий, которые попадают из $\Pi_{s}^{+}$ в $\Pi_{s}^{-}$не менее, чем за $\bar{k}_{s}$ итераций отображения $f$. В частности, это означает, что $\Pi_{s}^{+}$и $\Pi_{s}^{-}$содержат целиком полоски $\sigma_{k}^{0 s}$ и $\sigma_{k}^{1 s}$ с номерами $k \geqslant \bar{k}_{s}$ и не содержат полосок с номерами, меньшими $\bar{k}_{s}$. Более того, без ограничения обшности, $\Pi_{s}^{+}$и $\Pi_{s}^{-}$могут быть выбраны так, что

$$
\begin{aligned}
& \Pi_{1}^{+}=\left\{\left(x_{01}, y_{01}\right)|| x_{01}-\left.x_{1}^{+}\left|\leqslant \rho_{\bar{k}_{1}, \bar{k}_{2}},\right| y_{01}|\leqslant| \gamma_{1}\right|^{-\bar{k}_{1}}\left(y_{1}^{-}+\nu_{\bar{k}_{1}, \bar{k}_{2}}\right)\right\}, \\
& \Pi_{2}^{+}=\left\{\left(x_{02}, y_{02}\right)|| x_{02}-\left.x_{2}^{+}\left|\leqslant \nu_{\bar{k}_{1}, \bar{k}_{2}},\right| y_{02}|\leqslant| \gamma_{2}\right|^{-\bar{k}_{2}}\left(y_{2}^{-}+\rho_{\bar{k}_{1}, \bar{k}_{2}}\right)\right\}, \\
& \Pi_{1}^{-}=\left\{\left.\left(x_{11}, y_{11}\right)|| x_{11}|\leqslant| \lambda_{1}\right|^{\bar{k}_{1}}\left(x_{1}^{+}+\rho_{\bar{k}_{1}, \bar{k}_{2}}\right),\left|y_{11}-y_{1}^{-}\right| \leqslant \nu_{\bar{k}_{1}, \bar{k}_{2}}\right\}, \\
& \Pi_{2}^{-}=\left\{\left.\left(x_{12}, y_{12}\right)|| x_{12}|\leqslant| \lambda_{2}\right|^{\bar{k}_{2}}\left(x_{2}^{+}+\nu_{\bar{k}_{1}, \bar{k}_{2}}\right),\left|y_{12}-y_{2}^{-}\right| \leqslant \rho_{\bar{k}_{1}, \bar{k}_{2}}\right\},
\end{aligned}
$$

где

$$
\rho_{\bar{k}_{1}, \bar{k}_{2}}=L_{1} \sqrt{\left|\gamma_{1}\right|^{-\bar{k}_{1}}+\left|\lambda_{2}\right|^{\bar{k}_{2}}}, \quad \nu_{\bar{k}_{1}, \bar{k}_{2}}=L_{2}\left(\left|\gamma_{2}\right|^{-\bar{k}_{2}}+\left|\lambda_{1}\right|^{\bar{k}_{1}}\right),
$$

а $L_{1}$ и $L_{2}$ - положительные константы, не зависящие от $\bar{k}_{1}$ и $\bar{k}_{2}$.

Чтобы показать, что выбор именно таких окрестностей не ограничивает общности, возьмем, для определенности, в качестве первоначальных окрестностей $\Pi_{s}^{+}$ и $\Pi_{s}^{-}$малые квадраты с центром в точках $M_{s}^{+}$и $M_{s}^{-}$, соответственно, и со стороной длины $2 \varepsilon_{0}$. В силу того, что специальные окрестности не должны содержать точек, которые за меньшее, чем $\bar{k}_{s}$, число итераций отображения $f$ попадают из 
$\Pi_{s}^{+}$в $\Pi_{s}^{-}$, из (1.2) получаем, что при достаточно больших $\bar{k}_{1}$ и $\bar{k}_{2}$ окрестности $\Pi_{s}^{+}$ могут быть ужаты по координате $y$, a $\Pi_{s}^{-}-$по координате $x$ так, что

$$
\begin{array}{ll}
\left|y_{01}\right| \leqslant\left|\gamma_{1}\right|^{-\bar{k}_{1}}\left(y_{1}^{-}+\varepsilon_{0}\right), & \left|x_{11}\right| \leqslant\left|\lambda_{1}\right|^{\bar{k}_{1}}\left(x_{1}^{+}+\varepsilon_{0}\right), \\
\left|y_{02}\right| \leqslant\left|\gamma_{2}\right|^{-\bar{k}_{2}}\left(y_{2}^{-}+\varepsilon_{0}\right), & \left|x_{12}\right| \leqslant\left|\lambda_{2}\right|^{\bar{k}_{2}}\left(x_{2}^{+}+\varepsilon_{0}\right) .
\end{array}
$$

Так как в силу (1.3) $\bar{y}_{02}=c_{12} x_{11}+d_{12}\left(y_{11}-y_{1}^{-}\right)+\cdots$, а для координат $x_{11}$ и $\bar{y}_{02}$ требуется вьполнение оценок (1.6), то окрестность П ${ }_{1}^{-}$можно сузить так, чтобы координата $y_{11}$ удовлетворяла неравенству

$$
\left|y_{11}-y_{1}^{-}\right| \leqslant \max \left\{\frac{1}{\left|d_{12}\right|}\left(\left|\bar{y}_{02}\right|+\left|c_{12}\right|\left|x_{11}\right|\right)\right\} \leqslant C_{1}\left(\left|\gamma_{2}^{-\bar{k}_{2}}\right|+\left|\lambda_{1}^{\bar{k}_{1}}\right|\right) .
$$

В силу (1.4) $\bar{y}_{01}=c_{21} x_{12}+d_{21}\left(y_{12}-y_{2}^{-}\right)^{2}+\cdots$. Так как $d_{21} \neq 0$, то в силу (1.6) окрестность $\Pi_{2}^{-}$можно, в свою очередь, сузить так, чтобы координата $y_{12}$ удовлетворяла бы неравенству

$$
\left|y_{12}-y_{2}^{-}\right| \leqslant C_{2} \sqrt{\left|\gamma_{1}\right|^{-\bar{k}_{1}}+\left|\lambda_{2}\right|^{\bar{k}_{2}}}
$$

Теперь, в силу (1.3), (1.4) и (1.6)-(1.8) окрестности $\Pi_{1}^{+}$и $\Pi_{2}^{+}$можно еше сузить так, чтобы для координат $x_{01}$ и $x_{02}$ выполнялись оценки

$$
\left|x_{01}-x_{1}^{+}\right| \leqslant C_{3} \sqrt{\left|\gamma_{1}\right|^{-\bar{k}_{1}}+\left|\lambda_{2}\right|^{\bar{k}_{2}}}, \quad\left|x_{02}-x_{2}^{+}\right| \leqslant C_{4}\left(\left|\gamma_{2}\right|^{-\bar{k}_{2}}+\left|\lambda_{1}\right|^{\bar{k}_{1}}\right) .
$$

Если с полученными окрестностями гетероклинических точек провести еше раз такую же операцию, то получим соотношения (1.5).

Окрестности, определяемые формулой (1.5), будем называть специальнымми окрестностями точек $M_{1}^{+}, M_{2}^{+}, M_{1}^{-}$и $M_{2}^{+}$и именно их и будем рассматривать. Соответствуюшую специальную окрестность гетероклинического контура будем обозначать либо просто как $U$, либо как $U\left(\bar{k}_{1}, \bar{k}_{2}\right)$, если хотим подчеркнуть зависимость ее размеров от номеров полосок. Аналогично, и множество $N$, если необходимо, будем обозначать как $N\left(\bar{k}_{1}, \bar{k}_{2}\right)$.

1.4. Условия пересечений подков и полосок. Так как $\Gamma_{12}-$ траектория трансверсального пересечения многообразий $W^{u}\left(O_{1}\right)$ и $W^{s}\left(O_{2}\right)$, то пересечения любых полосок $T_{12} \sigma_{k}^{11}$ с любьми полосками $\sigma_{j}^{02}$ при достаточно больших $k$ и $j$ будут состоять из одной компоненты связности. Образы $T_{21}\left(\sigma_{j}^{12}\right)$ полосок $\sigma_{j}^{12}$ имеют вид подков, накапливающихся при $j \rightarrow \infty$ к "параболе" $T_{21}\left(W_{\mathrm{loc}}^{u}\left(O_{2}\right)\right) \subset$ $W^{u}\left(O_{2}\right) \cap \Pi_{1}^{+}$(рис. 2). Очевидно, траектории множества $N$ должны пересекать окрестность $\Pi_{1}^{+}$в точках пересечения подков $T_{21}\left(\sigma_{j}^{12}\right)$ и полосок $\sigma_{i}^{01}$ для всевозможных $i \geqslant \overline{k_{1}}$ и $j \geqslant \overline{k_{2}}$. Следовательно, структура множества $N$ сушественно зависит от геометрических свойств таких пересечений.

Будем говорить, что подкова $T_{21}\left(\sigma_{j}^{12}\right)$ имеет правильное пересечение с полоской $\sigma_{i}^{01}$, если: 1$)$ множество $T_{21}\left(\sigma_{j}^{12}\right) \cap \sigma_{i}^{01}$ непусто и состоит из двух компонент 


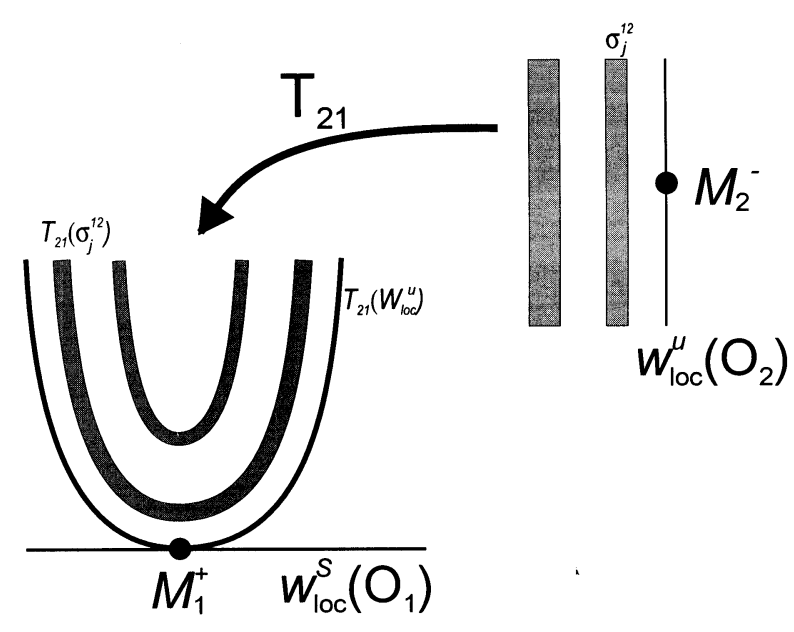

Рис. 2

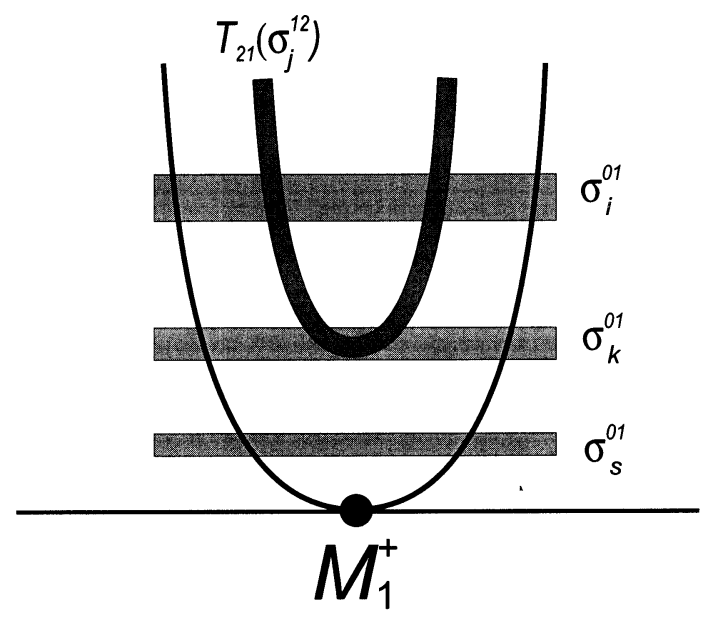

Рис. 3

связности; 2) отображения $T_{21}^{(1)} T_{02}^{j}$ и $T_{21}^{(2)} T_{02}^{j}$, определенные на $\sigma_{j}^{02}$ и имеющие области значений на соответствующих компонентах пересечений $T_{21}\left(\sigma_{j}^{12}\right) \cap \sigma_{i}^{01}$, являются седловьми в смысле [10] (грубо говоря, эти отображения являются растягивающими по координате $y_{02}$ и сжимающими по координате $x_{02}$ в $\Pi_{2}^{+}$).

Различные типы пересечений подковы $T_{21}\left(\sigma_{j}^{12}\right)$ с полосками на $\Pi_{1}^{+}$показаны на рис. 3. Подкова имеет правильное пересечение с полоской $\sigma_{i}^{01}$, неправильное - с полоской $\sigma_{k}^{01}$, и пустое - с полоской $\sigma_{s}^{01}$.

Аналогично [7], [8] доказывается следующая

Лемма 1. Существуют положсительная константа $S_{1}$ и достаточно 
больиие челье $\bar{k}_{1}$ и $\bar{k}_{2}$ такие, что если $i \geqslant \bar{k}_{1}, j \geqslant \bar{k}_{2} u$

1) если выполнено неравенство

$$
d_{21}\left(\gamma_{1}^{-i} y_{1}^{-}-c_{21} \lambda_{2}^{j} x_{2}^{+}\right)>S_{i j}\left(\bar{k}_{1}, \bar{k}_{2}\right)
$$

əде $S_{i j}=S_{1}\left(\left|\gamma_{1}\right|^{-i}+\left|\lambda_{2}\right|^{j}\right) \rho\left(\bar{k}_{1}, \bar{k}_{2}\right)$ u $\rho\left(\bar{k}_{1}, \bar{k}_{2}\right) \rightarrow 0$ nри $\bar{k}_{1}, \bar{k}_{2} \rightarrow \infty$, mo пересечение подковы $T_{21}\left(\sigma_{j}^{12}\right)$ с полоской $\sigma_{i}^{01}$ будет правильным;

2) если выполнено неравенство

$$
d_{21}\left(\gamma_{1}^{-i} y_{1}^{-}-c_{21} \lambda_{2}^{j} x_{2}^{+}\right)<-S_{i j}\left(\bar{k}_{1}, \bar{k}_{2}\right)
$$

$m o T_{21}\left(\sigma_{j}^{12}\right) \cap \sigma_{i}^{01}=\varnothing$

Из (1.10) и (1.11) вытекает, что неравенство

$$
d_{21}\left(\gamma_{1}^{-i} y_{1}^{-}-c_{21} \lambda_{2}^{j} x_{2}^{+}\right) \geqslant-S_{i j}\left(\bar{k}_{1}, \bar{k}_{2}\right)
$$

необходимо для того, чтобъ подкова $T_{21}\left(\sigma_{j}^{12}\right)$ имела непустое (возможсно $и$ неправильное) пересечение с полоской $\sigma_{i}^{01}$.

Неравенства (1.10)-(1.12) имеют весьма простой геометрический смысл. В силу (1.2) полоска $\sigma_{i}^{01}$ представляет собой узкий горизонтальный прямоугольник с "центральной" линией $y_{01}=\gamma_{1}^{-i} y_{1}^{-}$. Полоска $\sigma_{j}^{12}$ - это узкий вертикальный прямоугольник с “центральной” линией $x_{12}=\lambda_{2}^{j} x_{2}^{+}$. В силу (1.4) полоска $\sigma_{j}^{12}$ отображается относительно $T_{21}$ в подкову $T_{21}\left(\sigma_{j}^{12}\right)$, у которой парабола

$$
y_{01}=c_{21} \lambda_{2}^{j} x_{2}^{+}+\frac{d_{21}}{b_{21}^{2}}\left(x_{01}-x_{1}^{+}\right)^{2}
$$

является “центральной” линией. При вьполнении неравенства

$$
d_{21}\left(\gamma_{1}^{-i} y_{1}^{-}-c_{21} \lambda_{2}^{j} x_{2}^{+}\right)>0
$$

прямая $y_{01}=\gamma_{1}^{-i} y_{1}^{-}$и парабола пересекаются в двух точках. При

$$
d_{21}\left(\gamma_{1}^{-i} y_{1}^{-}-c_{21} \lambda_{2}^{j} x_{2}^{+}\right)<0
$$

они не имеют пересечений, а при

$$
d_{21}\left(\gamma_{1}^{-i} y_{1}^{-}-c_{21} \lambda_{2}^{j} x_{2}^{+}\right)=0
$$

касаются. Коэффициент $S_{i j}\left(\bar{k}_{1}, \bar{k}_{2}\right)$ в (1.10)-(1.12) учитывает ненулевую толщину полоски и подковы. 


\section{§2. Кодировки и нетривиальные гиперболические подмножества}

Удобный способ описания структуры множества $N$ состоит в построении кодировок его траекторий. Множества полосок $\sigma_{k}^{01}$ на $\Pi_{1}^{+}, \sigma_{k}^{11}$ на $\Pi_{1}^{-}, \sigma_{k}^{02}$ на $\Pi_{2}^{+}$и $\sigma_{k}^{12}$ на П 2 обозначим, соответственно, через $\sigma_{01}, \sigma_{11}, \sigma_{02}$ и $\sigma_{12}$. Пусть $\Lambda \in N$. Предположим, что траектория $\Lambda$ не является асимптотической к $O_{1}$ и $O_{2}$. Тогда она пересекает окрестности $\Pi_{1}^{+}, \Pi_{1}^{-}, \Pi_{2}^{+}$и $\Pi_{2}^{-}$только по точкам, принадлежащим полоскам из множеств $\sigma_{01}, \sigma_{11}, \sigma_{02}$ и $\sigma_{12}$, соответственно. Пусть $\left(\ldots, M_{\alpha \beta}^{-s}, \ldots, M_{\alpha \beta}^{s}, \ldots\right)$, $\alpha=0,1, \beta=1,2,-$ последовательные точки пересечения траектории $\Lambda$ с полосками из множеств $\sigma_{\alpha \beta}$. Очевидно, для этих точек должны выполняться соотношения

$$
\begin{aligned}
& M_{01}^{s} \in \sigma_{k_{s}(1)}^{01} \subset \Pi_{1}^{+}, \\
& M_{11}^{s}=T_{01}^{k_{s}(1)}\left(M_{01}^{s}\right) \in \sigma_{k_{s}(1)}^{11} \subset \Pi_{1}^{-}, \\
& M_{02}^{s}=T_{12}\left(M_{11}^{s}\right) \equiv f^{n_{1}}\left(M_{11}^{s}\right) \in \sigma_{k_{s}(2)}^{02} \subset \Pi_{2}^{+}, \\
& M_{12}^{s}=T_{02}^{k_{s}(2)}\left(M_{02}^{s}\right) \in \sigma_{k_{s}(2)}^{12} \subset \Pi_{2}^{-}, \\
& M_{01}^{s+1}=T_{21}\left(M_{21}^{s}\right) \equiv f^{n_{2}}\left(M_{12}^{s}\right) \in \sigma_{k_{s+1}(1)}^{01} \subset \Pi_{1}^{+},
\end{aligned}
$$

$s=0, \pm 1, \ldots$, г де, напомним, $n_{1}$ и $n_{2}$ такие натуральные числа, что $f^{n_{1}}\left(M_{1}^{-}\right)=M_{2}^{+}$, $f^{n_{2}}\left(M_{2}^{-}\right)=M_{1}^{+}$.

В соответствии с (2.1) кодировку траектории $\Lambda$ определим как бесконечную последовательность из двух символов $\{1,2\}$

$$
(\ldots, 2,2, \overbrace{1,1, \ldots, 1}^{k_{s}(1)+n_{1}}, \overbrace{2,2, \ldots, 2}^{k_{s}(2)+n_{2}}, 1,1 \ldots)
$$

в которой длины следующих друг за другом полных отрезков, составленных из символов “1” и “2”, равны, соответственно, $\left(k_{s}(1)+n_{1}\right)$ и $\left(k_{s}(2)+n_{2}\right)$. Другими словами, если точка траектории $\Lambda$ попала в окрестность $\Pi_{1}^{+}$, то в последовательности (2.2) этой точке будет отвечать символ “1". Далее символ "1" будет отвечать каждой последующей точке траектории $\Lambda$ до тех пор, пока очередная ее точка не попадет в окрестность $\Pi_{2}^{+}$. Этой последней точке поставим в соответствие уже символ "2". Точкам последующих итераций будем ставить в соответствие символ “ 2 " до тех пор, когда очередная точка траектории $\Lambda$ снова попадет в окрестность $\Pi_{1}^{+}$. Этой последней точке поставим в соответствие символ “1” и т. д.

Кодировки вида (2.2) можно распространить и на асимптотические к $O_{1}$ и $\mathrm{O}_{2}$ траектории из $N$. При этом траектория $O_{1}$ будет иметь кодировку $(\ldots, 1, \ldots, 1, \ldots)$, траектория $O_{2}-$ кодировку $(\ldots, 2, \ldots, 2, \ldots)$, траектория $\Gamma_{12}-$ кодировку $(\ldots, 1, \ldots 1,2, \ldots, 2, \ldots)$, траектория $\Gamma_{21}$ - кодировку $(\ldots, 2, \ldots, 2,1, \ldots, 1 \ldots)$. Траектории, $\alpha$-предельной (соответственно, $\omega$-предельной) к $O_{1}$, будет отвечать кодировка вида $(2.2)$, имеющая бесконечную последовательность из символов " 1 ”, стоящую на левом (соответственно, на правом) конце. Аналогично, $\alpha(\omega)$-предельной к $O_{2}$ траектории из $N$ будет отвечать кодировка вида (2.2), имеюшая бесконечную последовательность из символов “2”, стоящую на левом (правом) конце. 
Если бы $\Gamma_{21}$ была грубой гетероклинической траекторией (т.е. многообразия $W^{u}\left(O_{2}\right)$ и $W^{s}\left(O_{1}\right)$ пересекались бы по ней трансверсально), то мы имели бы взаимно однозначное соответствие между множеством траекторий $N$ (которые все были бы в этом случае седлового типа) и множеством указанных кодировок [10]. В нашем случае, когда $\Gamma_{21}$ - негрубая гетероклиническая траектория, это уже не так.

Во-первых, заметим, что в $N$ не может быть траекторий с такими кодировками, у которых для некоторого $s$ числа $j=k_{s}(2)$ и $i=k_{s+1}(1)$ удовлетворяют неравенству (1.11), поскольку в этом случае $T_{21}\left(\sigma_{j}^{12}\right) \cap \sigma_{i}^{01}=\varnothing$ в силу леммы 1. Во-вторых, даже если мы ограничимся только такими кодировками, у которых для всех $s=0, \pm, 1, \ldots$, числа $k_{s}(2)$ и $k_{s+1}(1)$ удовлетворяют неравенству (1.10) с $j=k_{s}(2), i=k_{s+1}(1)$, то взаимно однозначного соответствия, как в грубом случае, не будет. Точнее, здесь имеет место следуюшая

ТЕОРема 1. Для произвольной кодировки вида $(2.2)$, в которой $\infty>k_{s}(1)$ $\geqslant \bar{k}_{1}, \infty>k_{s}(2) \geqslant \bar{k}_{2}$, и для любого $s=0, \pm 1, \ldots$ числа $k_{s}(2)$ и $k_{s+1}(1)$ удовлетворяют неравенству (1.10) с $j=k_{s}(2), i=k_{s+1}(1)$, в $N$ существует континуум траекторий седлового типа, каждая из которых имеет данную кодировку. Множество таких траекторий находится во взаимно однозначном соответствии с множеством бесконечных в обе стороны последовательностей, составленных из двух символов.

Эта теорема доказывается аналогично [7], [8] и имеет простое геометрическое объяснение. Именно, неравенство (1.10) гарантирует, что пересечение подковы $T_{21} \sigma_{j}^{12}$ и полоски $\sigma_{i}^{01}$ правильное и состоит из двух компонент связности, которые обозначим как $\Delta_{i j}^{3}$ и $\Delta_{i j}^{4}$. Ясно, что траектории из $N$ с одной и той же кодировкой вида (2.2), но имеюшие точки пересечения с разными такими компонентами связности, нужно различать. Именно, более точную по сравнению с (2.2) кодировку будет представлять следуюшая бесконечная в обе стороны последовательность

$$
(\ldots, 1,1, \overbrace{2,2, \ldots, 2, \alpha_{s}}^{k_{s}(2)+n_{2}}, \overbrace{1,1, \ldots, 1}^{k_{s+1}(1)+n_{1}}, \overbrace{2,2, \ldots, 2, \alpha_{s+1}}^{k_{s+1}(2)+n_{2}}, 1,1, \ldots),
$$

составленная уже из четырех символов $\{$ “" 1 , “2”, " 3 ”, “4” $\}$, где символ $\alpha_{s}-$ это либо “ 3 ”, либо “4”, в зависимости от того, принадлежит компоненте $\Delta_{i j}^{3}$ или компоненте $\Delta_{i j}^{4}$ полоски $\sigma_{i}^{01}$ соответствуюшая точка траектории из $N$ (здесь $i=k_{s+1}(1)$, $\left.j=k_{s}(2)\right)$.

Отображение $T_{21} T_{02}^{j}: \sigma_{j}^{02} \rightarrow \Delta_{i j}^{\alpha} \subset \sigma_{i}^{01}$ является седловым по определению правильного пересечения. Седловым будет также отображение $T_{12} T_{01}^{i}: \Delta_{i j}^{\alpha} \rightarrow \sigma_{k}^{02}$ для любого $k \geqslant \bar{k}_{2}$, поскольку $\Gamma_{12}-$ грубая гетероклиническая траектория. Зафиксируем последовательность $\left\{\alpha_{s}\right\}, s=0, \pm 1, \ldots$, составленную из символов “3" и “4”. Тогда по лемме о седловой неподвижной точке в счетном произведении пространств [10] бесконечной последовательности таких отображений, строяшейся в соответствии с (2.3), будет отвечать единственная траектория, не покидающая окрестность $U$ контура $C$. Множество таких траекторий, отвечающих различным последовательностям $\left\{\alpha_{s}\right\}$, будет иметь мощность континуум. 


\section{§3. Классы двумерных диффеоморфизмов с негрубым гетероклиническим контуром}

Заметим, что координаты на $U_{1}$ и $U_{2}$ всегда можно выбрать так, что $x_{2}^{+}$и $y_{1}^{-}$будут положительны. Тогда структура множеств решений неравенств (1.10)-(1.12) будет зависеть, в первую очередь, от знаков величин $\lambda_{2}, \gamma_{1}, c_{21}$ и $d_{21}$. На рис. 4 в случае $\lambda_{s}>0, \gamma_{s}>0, s=1,2$, представлено четыре типа негрубых гетероклинических контуров.

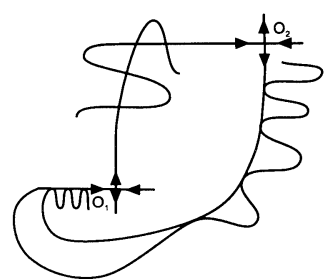

a)

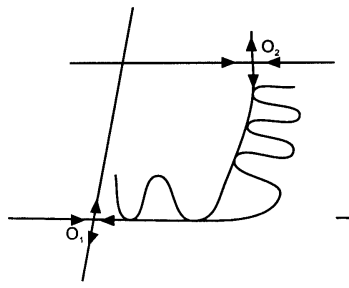

B)

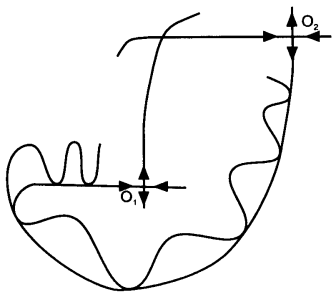

б)

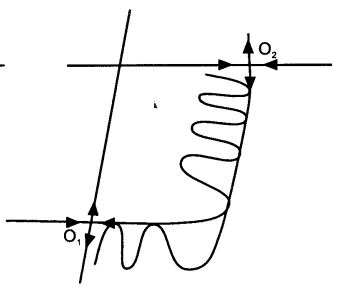

г)

Рис. 4

Наиболее простая структура множества решений неравенств (1.10)-(1.12) будет в случае диффеоморфизмов, представленњых на рис. 4а) и 4б) и отвечающих следуюшей комбинации знаков: $\lambda_{2}>0, \gamma_{1}>0, c_{21}<0$.

Диффеоморфизмы, у которых при этом $d_{21}<0$ (рис. 4а), отнесем к первому классу. Легко видеть, что в этом случае для любых $i \geqslant \bar{k}_{1}, j \geqslant \bar{k}_{2}$ всегда будет выполняться неравенство (1.11), т.е. $T_{21}\left(\sigma_{j}^{12}\right) \cap \sigma_{i}^{01}=\varnothing$ при всех достаточно больших $i$ и $j$. Более того, подковы $T_{21}\left(\sigma_{j}^{12}\right)$ и полоски $\sigma_{i}^{01}$ будут лежать на $\Pi_{1}^{+}$по разные стороны от $W_{\mathrm{loc}}^{s}\left(O_{1}\right)$. Здесь справедлива следуюшая

Tеорема 2 [11]. Пусть $f$-диффеоморфизм первого класса. Тогда существует такая окрестность $U$ гетероклинического контура $C$, что множество $N$ траекторий, не покидающих $U$, имеет тривиальную структуру: $N=$ $O_{1} \cup O_{2} \cup \Gamma_{12} \cup \Gamma_{21}$.

Диффеоморфизмы с негрубым гетероклиническим контуром, у которых $\lambda_{2}>0$, $\gamma_{1}>0, c_{21}<0, d_{21}>0$ (рис. $4 \sigma$ ), отнесем ко второму классу. Заметим, что 
здесь при достаточно больших $\bar{k}_{1}$ и $\bar{k}_{2}$ всегда будет выполняться уже неравенство $(1.10)$, т.е. полоски $\sigma_{i}^{01}$ и подковы $T_{21}\left(\sigma_{j}^{12}\right)$ при любых $i \geqslant \bar{k}_{1}$ и $j \geqslant \bar{k}_{2}$ будут иметь правильные пересечения. Тогда по теореме 1 все траектории множества $N$, за исключением $\Gamma_{21}$, будут седловыми. При этом утверждение теоремы 1 здесь можно усилить, указав топологически сопряженную $\left.f\right|_{N}$ систему.

Рассмотрим топологическую схему Бернулли из четырех символов $\{1,2,3,4\}$ и отождествим в ней траектории

$$
(\ldots, 2, \ldots, 2,3,1, \ldots, 1, \ldots) \text { и }(\ldots, 2, \ldots, 2,4,1, \ldots, 1, \ldots)
$$

в одну, которую обозначим через $\widetilde{\omega}$. У полученной фактор-системы рассмотрим подсистему $\Omega$ траекторий, удовлетворяющих следующим условиям:

2.1) $\Omega$ содержит траектории $(\ldots, 1, \ldots, 1, \ldots),(\ldots, 2, \ldots, 2, \ldots)$ и $\widetilde{\omega}$;

2.2 за символом "1" не может идти символ "3" или "4", а за символом "2" символ "1"; за символом " 3 " или "4" обязательно идет символ "1";

2.3) длина любого полного отрезка, составленного из символа "1", не меньше чем $\bar{k}_{1}+n_{1}$, а из символа "2" - не меньше чем $\bar{k}_{2}+n_{2}-1$.

ТЕОРема 3. Пусть $f$-диффеоморфизм второго класса. Тогда существуют такие $\bar{k}_{1}, \bar{k}_{2}, n_{1}$ и $n_{2}$, что $\left.f\right|_{N}$ будет сопряжено с дискретной динамической системой $\Omega$.

Остальные диффеоморфизмы с негрубым гетероклиническим контуром отнесем к третьему классу (таковыми являются, например, диффеоморфизмы, представленные на рис. 4в) и 4г)). У таких диффеоморфизмов в силу теоремы 1 сушествуют нетривиальные гиперболические подмножества, структуру которых можно также описать в терминах топологической сопряженности. Именно, рассмотрим подсистему $\widetilde{\Omega} \subset \Omega$, удовлетворяюшую условиям 2.1)-2.3) и условию

$2.4)$ Пусть $k_{s}(2)+n_{2}-1$ и $k_{s+1}(1)+n_{1}$ - длины следующих друг за другом полных отрезков, составленных из символов "2" и "1". Тогда для любого $s$ числа $j=k_{s}(2)$ и $i=k_{s+1}(1)$ удовлетворяют неравенству (1.10).

Аналогично [7], [8], из теоремы 1 выводится следующая

Tеорема 4. Пусть $f$-диффеоморфизм третьего класса. Тогда в $N$ можно указать подсистему $\widetilde{N}$ такую, что, во-первых, $\left.f\right|_{\widetilde{N}}$ сопряжена с $\widetilde{\Omega}, u$, во-вторых, все траектории подсистемы $\widetilde{N}$, за исключением $\Gamma_{21}$, седлового muna.

\section{§4. Модули $\Omega$-сопряженности}

Из теорем 2 и 3 вытекает, что для диффеоморфизмов первого и второго классов структура множества $N$ не меняется при переходе от одной системы к другой на соответствуюшей бифуркационной поверхности диффеоморфизмов с негрубым гетероклиническим контуром. Для диффеоморфизмов третьего класса это будет уже не так. Обозначим через $H_{3}$ локально связную бифуркационную поверхность коразмерности один в пространстве динамических систем, отвечающую диффеоморфизмам третьего класса. Мы покажем, что для систем в $H_{3}$ существуют непрерывные инварианты (модули) $\Omega$-сопряженности. 
Напомним определение модуля.

ОПРЕДЕЛЕНИЕ 1 [4], [5]. Будем говорить, что система $f$ имеет модуль, если в пространстве динамических систем $f$ лежит в некотором банаховом многообразии $\mathscr{M}$, на котором определен непрерывный локально непостоянный функционал $h$ со следующим свойством: если $f_{1}, f_{2} \in \mathscr{M}$ и $f_{1}$ и $f_{2}$ эквивалентны, то $h\left(f_{1}\right)=$ $h\left(f_{2}\right)$. Система $f$ uмеeт $m$ модулей, если $f$ лежит в некотором банаховом многообразии, на котором существует $m$ независимых модулей. Наконец, говорят, что $f$ имеет счетное мнохсество модулей, если $f$ имеет $m$ модулей для любого наперед заданного $m$.

В теории динамических систем наиболее употребительны модули топологической и $\Omega$-эквивалентности (топологической эквивалентности на множестве неблуждающих траекторий).

Как мы отмечали, топологические модули для систем с негрубыми гетероклиническими траекториями ввел Пэлис [1]. Аналогичные модули имеют место и для систем с негрубыми гомоклиническими траекториями. Но ситуация здесь оказывается более сложной. Именно, как показано в [3], [4], диффеоморфизмы третьего класса [7] с негрубой гомоклинической траекторией обладают уже модулями $\Omega$-сопряженности, и более того, на бифуркационной поверхности таких систем плотны системы со счетным множеством $\Omega$-модулей [5], [6]. ${ }^{1}$ В некотором смысле аналогичная ситуация будет иметь место и для систем с негрубым гетероклиническим контуром, что мы покажем ниже.

Из [1] непосредственно вытекает, что инвариант

$$
\theta=-\frac{\ln \left|\lambda_{2}\right|}{\ln \left|\gamma_{1}\right|}
$$

является модулем топологической сопряженности для диффеоморфизмов с негрубым гетероклиническим контуром. Однако, если ограничиться только рассмотрением условий $\Omega$-сопряженности, то диффеоморфизмы первого и второго классов в силу теорем 2 и 3 на соответствующих бифуркационных поверхностях оказываются грубыми. В то же время, диффеоморфизмы третьего класса будут обладать модулями $\Omega$-сопряженности.

Пусть $U$ и $U^{\prime}$ - некоторые окрестности гетероклинических контуров $C$ и $C^{\prime}$ диффеоморфизмов $f$ и $f^{\prime}$. Пусть $\Omega(f)$ и $\Omega\left(f^{\prime}\right)$ - множества неблуждаюших траекторий, целиком лежащих в $U$ и $U^{\prime}$, соответственно. Заметим, что $\Omega(f)$ не всегда совпадает с $N$ (именно, $\Omega(f) \subseteq N$ ), но во всяком случае нетривиальное множество $\widetilde{N}$ (из теоремы 4) содержится в $\Omega(f)$.

ОПРЕДЕЛЕНИЕ 2. Будем Говорить, что $f$ и $f^{\prime}-\Omega$-сопряжсень, если существует гомеоморфизм $h: \Omega(f) \rightarrow \Omega\left(f^{\prime}\right)$ такой, что $h\left(O_{s}\right)=O_{s}^{\prime}, s=1,2, h\left(\Gamma_{12}\right)=\Gamma_{12}^{\prime}$,

\footnotetext{
${ }^{1}$ Отметим также, что для систем с гомоклинической петлей состояния равновесия типа седло-фокус величина $\rho$ (седловой индекс), введенная в [12], является топологическим модулем. Для трехмерных систем это установлено в [13], [14]. Но, как следует из [16], [17], в случае, когда в окрестности гомоклинической петли имеет место сложное поведение траекторий [12], [15], инвариант $\rho$ является уже модулем $\Omega$-эквивалентности.
} 
$h\left(\Gamma_{21}\right)=\Gamma_{21}^{\prime}$ и следуюшая диаграмма

коммутативна.

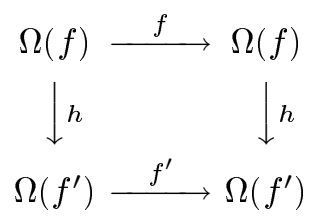

Прежде всего укажем некоторые свойства $\Omega$-сопряженных диффеоморфизмов $f$ и $f^{\prime}$, вытекаюшие из данного определения.

Лемма 2. Пусть $f, f^{\prime} \in H_{3}, u f u f^{\prime}-\Omega$-сопряжены в некоторых окрестностях $U$ и $U^{\prime}$ гетероклинических контуров $C$ и $C^{\prime}, u h$ - сопрягающий гомеоморфизм. Тогда существуют такие специальные окрестности $V \subseteq U$ и $V^{\prime} \subseteq U^{\prime}$, ито

1) $h(V \cap \Omega(f))=V^{\prime} \cap \Omega\left(f^{\prime}\right)$;

2) гомеоморфизм $h: \Omega\left(\left.f\right|_{V}\right) \rightarrow \Omega\left(\left.f^{\prime}\right|_{V^{\prime}}\right)$ сохраняет кодировки вида (2.2).

ДокаЗАТЕЛЬСтво. Пусть гетероклинические точки $M_{s}^{+}, M_{s}^{-}$и $M_{s}^{\prime+}, M_{s}^{\prime-}$, $s=1,2$, выбраны так, что

$$
h\left(M_{s}^{+}\right)=M_{s}^{\prime+}, h\left(M_{s}^{-}\right)=M_{s}^{\prime-} .
$$

Такие точки будем называть сопрязсенными. Для диффеоморфизма $f$ рассмотрим специальную окрестность $V \equiv V\left(\bar{k}_{1}, \bar{k}_{2}\right)$. В силу непрерывности $h$ и условия (4.1) при достаточно больших $\bar{k}_{1}$ и $\bar{k}_{2}$ мы получаем, что $h\left(\Omega\left(\left.f\right|_{V}\right)\right) \subset U^{\prime}$. В силу коммутативности диаграммы и опять же условия (4.1) можно выбрать такую специальную окрестность $V^{\prime} \equiv V^{\prime}\left(\bar{k}_{1}, \bar{k}_{2}\right) \subseteq U^{\prime}$, что $h\left(\Omega\left(\left.f\right|_{V}\right)\right) \subset V^{\prime}$. Наконец, гомеоморфизм $h: \Omega\left(\left.f\right|_{V}\right) \rightarrow \Omega\left(\left.f^{\prime}\right|_{V^{\prime}}\right)$ сохраняет кодировки вида $(2.2)$ в силу коммутативности диаграммы. Это завершает доказательство леммы.

Далее будем считать, что $U \equiv V, U^{\prime} \equiv V^{\prime}$. В силу леммы 2 кодировки вида 2.2 соответствуюших траекторий из $\Omega(f)$ и $\Omega\left(f^{\prime}\right)$ должны совпадать. В частности, поскольку $\widetilde{N} \subset \Omega(f)$, то в $\Omega\left(f^{\prime}\right)$ должно содержаться множество траекторий, кодировки которых совпадают с кодировками траекторий из $\widetilde{N}$. Очевидно также, что не могут быть $\Omega$-сопряжены диффеоморфизм, представленный на рис. 4в), и диффеоморфизм, представленный на рис. 4г). Например, это следует из того, что любой диффеоморфизм такого типа, как на рис. 4в), не имеет траекторий, гомоклинических к $O_{1}$, тогда как очевидно, что у диффеоморфизма такого типа, как на рис. 4г), таких траекторий счетное множество. ${ }^{2}$

Далее будем изучать условия $\Omega$-сопряженности, связанные с модулями. Для определенности, все дальнейшие доказательства будем проводить для случая диффеоморфизмов такого же типа, как на рис. 4в), т.е. будем считать, что $\lambda_{1}>0$, $\gamma_{2}>0, c_{21}>0, d_{21}>0$. Доказательства представленных ниже утверждений в остальных случаях диффеоморфизмов третьего класса проводятся вполне аналогично.

Прежде всего установим, что величина $\theta$ является модулем $\Omega$-сопряженности диффеоморфизмов третьего класса, т.е. имеет место следуюшая

\footnotetext{
${ }^{2}$ Более подробно об условиях $\Omega$-сопряженности см., например, в [3] , [18].
} 
ТеОРема 5. Пусть $f, f^{\prime} \in H_{3}, u f u f^{\prime}-\Omega$-сопряжены в некоторых окрестностях $U$ и $U^{\prime}$ гетероклинических контуров $C$ и $C^{\prime}$. Тогда $\theta=\theta^{\prime}$.

ДокАЗАтЕльство. Предположим, что $f$ и $f^{\prime}-\Omega$-сопряжены в некоторых окрестностях $U$ и $U^{\prime}$ гетероклинических контуров, но $\theta>\theta^{\prime}$. Пусть $M_{s}^{+}, M_{s}^{-}$и $M_{s}^{\prime+}, M_{s}^{\prime-}, s=1,2,-$ пары сопряженных гетероклинических точек.

Для $f$ рассмотрим множество пар $(i, j)$ натуральных чисел, удовлетворяющих неравенству (1.10), т.е. таких $i$ и $j$, что подкова $T_{21}\left(\sigma_{j}^{12}\right)$ пересекает полоску $\sigma_{i}^{01}$ правильно. Если прологарифмировать неравенство (1.10), то получим следующее:

$$
i<j \theta-\tau-S_{2}\left(\bar{k}_{1}, \bar{k}_{2}\right)
$$

где $S_{2}\left(\bar{k}_{1}, \bar{k}_{2}\right) \rightarrow 0$ при $\bar{k}_{1}, \bar{k}_{2} \rightarrow \infty$, и

$$
\tau=\frac{1}{\ln \left|\gamma_{1}\right|} \ln \frac{\left|c_{21} x_{2}^{+}\right|}{\left|y_{1}^{-}\right|}
$$

Для $f^{\prime}$ рассмотрим множество пар $(i, j)$ натуральных чисел, удовлетворяющих неравенству (1.12), т.е. такому

$$
i \leqslant j \theta^{\prime}-\tau^{\prime}+S_{2}^{\prime}\left(\bar{k}_{1}, \bar{k}_{2}\right)
$$

Заметим, что неравенству (4.4) удовлетворяют, во всяком случае, все такие числа $i$ и $j$, для которых $T_{21}^{\prime}\left(\sigma_{j}^{12}\right) \cap \sigma_{i}^{01} \neq \varnothing$.

Наконец, рассмотрим множество пар $(i, j)$ натуральных чисел, удовлетворяющих неравенству (4.2), но не удовлетворяющих неравенству (4.4). Для таких $i$ и $j$ вьполнено неравенство

$$
j \theta^{\prime}-\tau^{\prime}+\cdots<i<j \theta-\tau+\cdots
$$

где многоточиями обозначены члены, стремящиеся к нулю при $\bar{k}_{1}, \bar{k}_{2} \rightarrow \infty$. Неравенство (4.5) при достаточно больших $\bar{k}_{1}$ и $\bar{k}_{2}$ имеет счетное множество целочисленных решений, поскольку $\theta>\theta^{\prime}$ по предположению. Пусть $i=i^{*}, j=j^{*}$ одно из таких решений. Тогда, поскольку $i=i^{*}, j=j^{*}$ удовлетворяют неравенству (4.2), то у диффеоморфизма $f$ в силу теоремы 1 сушествуют неблуждающие траектории (седлового типа), имеющие кодировки вида $(2.2)$, в которых $k_{s}(2)=j^{*}$, $k_{s+1}(1)=i^{*}$ для некоторого $s$. В частности, такой кодировкой обладает однообходная периодическая траектория, имеющая по одной точке пересечения с полосками $\sigma_{i^{*}}^{01}$ и $\sigma_{j^{*}}^{02}$. При этом ее точка пересечения с полоской $\sigma_{i *}^{01}$ является неподвижной для отображения $T_{21} T_{02}^{j^{*}} T_{12} T_{01}^{i^{*}}: \sigma_{i^{*}}^{01} \rightarrow \sigma_{i^{*}}^{01}$, которое в данном случае аналогично известному отображению подковы Смейла. С другой стороны, числа $i=i^{*}, j=j^{*}$ не удовлетворяют неравенству (4.4), и поэтому для $f^{\prime}$ имеем, что $T_{21}^{\prime}\left(\sigma_{j^{*}}^{12}\right) \cap \sigma_{i^{*}}^{01}=\varnothing$. Таким образом, у $f^{\prime}$ вообще не может быть траекторий с кодировкой, в которой были бы соседние символы $k_{s}(2)=j^{*}, k_{s+1}(1)=i^{*}$. Следовательно, $f$ и $f^{\prime}$ не могут быть $\Omega$-сопряжены. Получили противоречие с первоначальным предположением, что $\theta>\theta^{\prime}$. Случай $\theta<\theta^{\prime}$ рассматривается аналогично, здесь достаточно поменять местами $f$ и $f^{\prime}$. Тем самьм, теорема доказана. 
Введем следующую величину

$$
\tau_{0} \equiv\left[\tau-\left(n_{1}+n_{2}\right)\right] \quad(\bmod (1+\theta))
$$

где $n_{1}$ - число итераций отображения $f$, связывающих точки $M_{1}^{-}$и $M_{2}^{+}$траектории $\Gamma_{12}$, а $n_{2}$ - число итераций отображения $f$, связываюших точки $M_{2}^{-}$и $M_{1}^{+}$ траектории $\Gamma_{21}$. Ниже мы покажем, что $\tau_{0}$ является модулем $\Omega$-сопряженности диффеоморфизмов третьего класса при иррациональном $\theta$.

Аналогично [4, лемма 1.5] доказывается, что величина $\tau$ (а следовательно, и $\left.\tau_{0}\right)$ не зависит от гладких замен координат на $U_{1}$ и $U_{2}$, сохраняющих вид (1.1) отображений $T_{01}$ и $T_{02}$. Но величина $\tau$ зависит, вообще говоря, от того, какие пары гетероклинических точек траекторий $\Gamma_{12}$ и $\Gamma_{21}$ выбираются. Это уже не так для $\tau_{0}$, именно, имеет место следующая

ЛЕмма 3. Величина $\tau_{0}$ не зависит от выбора в $U_{1}$ и $U_{2}$ пар гетероклинических точек траекторий $\Gamma_{12} u \Gamma_{21}$.

ДокаЗАтЕльство. Рассмотрим пары точек $\widetilde{M}_{1}^{-} \equiv T_{01}^{-1} M_{1}^{-}$и $M_{2}^{+}$траектории $\Gamma_{12}$, и $M_{2}^{-}$и $M_{1}^{+}$траектории $\Gamma_{21}$. Очевидно, при таком выборе гетероклинических точек из всех параметров, входящих в формулу (4.3) для $\tau$, изменится только координата " $y$ ” новой гетероклинической точки $\widetilde{M}_{1}^{-}$: она будет в силу (1.1) равна $\gamma_{1}^{-1} y_{1}^{-}$. Из (4.3) тогда получим для $\widetilde{\tau} \equiv \tau\left(M_{1}^{+}, \widetilde{M}_{1}^{-}, M_{2}^{+}, M_{2}^{-}\right)$следуюшее значение

$$
\widetilde{\tau}=\frac{1}{\ln \left|\gamma_{1}\right|} \ln \frac{\left|c_{21} x_{2}^{+}\right|}{\left|\gamma_{1}^{-1} y_{1}^{-}\right|}=\tau+1
$$

Так как число итераций отображения $f$, связывающих точки $\widetilde{M}_{1}^{-}$и $M_{2}^{+}$, станет равным $\left(n_{1}+1\right)$, то из (4.6) получаем, что

$$
\widetilde{\tau}_{0}=\left[\tau+1-\left(n_{1}+1+n_{2}\right)\right] \quad(\bmod (1+\theta))=\tau_{0} .
$$

Рассмотрим такие пары гетероклинических точек: $M_{1}^{-}, \widetilde{M}_{2}^{+}=T_{02} M_{2}^{+}$и $M_{2}^{-}$и $M_{1}^{+}$. Координата “ $x$ ” точки $\widetilde{M}_{2}^{+}$в силу (1.1) равна $\lambda_{2} x_{2}^{+}$, и, следовательно, из (4.3) и (4.6) получаем, что

$$
\begin{aligned}
\widetilde{\tau} & =\frac{1}{\ln \left|\gamma_{1}\right|} \ln \left|\frac{c_{21} \lambda_{2} x_{2}^{+}}{y_{1}^{-}}\right|=\tau-\theta, \\
\widetilde{\tau}_{0} & =\left[\tau-\theta-\left(n_{1}+1+n_{2}\right)\right](\bmod (1+\theta))=\tau_{0} .
\end{aligned}
$$

Рассмотрим теперь такие пары гетероклинических точек: $M_{1}^{-}, M_{2}^{+}$и $\widetilde{M}_{2}^{-} \equiv T_{02}^{-1} M_{2}^{-}, M_{1}^{+}$. В этом случае роль $T_{21}$ будет играть отображение $T_{21} T_{02}: T_{02}^{-1}\left(\Pi_{2}^{-}\right) \rightarrow \Pi_{1}^{+}$, которое в силу (1.1) можно представить в следуюшем виде

$$
\begin{gathered}
\bar{x}_{01}-x_{1}^{+}=a_{21} \lambda_{2} x_{12}^{\prime}+b_{21} \gamma_{2}\left(y_{12}^{\prime}-\gamma_{2}^{-1} y_{2}^{-}\right)+\cdots, \\
\bar{y}_{01}=c_{21} \lambda_{2} x_{21}^{\prime}+d_{21} \gamma_{2}^{2}\left(y_{12}^{\prime}-\gamma_{2}^{-1} y_{2}^{-}\right)^{2}+\cdots
\end{gathered}
$$


где $\left(x_{12}^{\prime}, y_{12}^{\prime}\right) \in T_{02}^{-1}\left(\Pi_{2}^{-}\right)$. В силу (4.3) и (4.6) получаем, что

$$
\begin{aligned}
\widetilde{\tau} & =\frac{1}{\ln \left|\gamma_{1}\right|} \ln \left|\frac{\lambda_{2} c_{21} x_{2}^{+}}{y_{1}^{-}}\right|=\tau-\theta, \\
\widetilde{\tau}_{0} & =\left[\tau-\theta-\left(n_{1}+n_{2}+1\right)\right](\bmod (1+\theta))=\tau_{0} .
\end{aligned}
$$

Рассмотрим, наконец, такие пары точек: $M_{1}^{-}, M_{2}^{+}$и $M_{2}^{-}, \widetilde{M}_{1}^{+} \equiv T_{01} M_{1}^{+}$. В этом случае роль $T_{21}$ будет играть уже отображение $T_{01} T_{21}: \Pi_{2}^{-} \rightarrow T_{01}\left(\Pi_{1}^{+}\right)$, которое в силу (1.1) записывается в виде

$$
\begin{gathered}
\bar{x}_{01}^{\prime}-\lambda_{1} x_{1}^{+}=a_{21} \lambda_{1} x_{12}+b_{21} \lambda_{1}\left(y_{12}-y_{2}^{-}\right)+\cdots \\
\bar{y}_{01}^{\prime}=\gamma_{1} c_{21} x_{21}+d_{21} \gamma_{1}\left(y_{12}-y_{2}^{-}\right)^{2}+\cdots
\end{gathered}
$$

Таким образом,

$$
\begin{aligned}
\widetilde{\tau} & =\frac{1}{\ln \left|\gamma_{1}\right|} \ln \left|\frac{\gamma_{1} c_{21} x_{2}^{+}}{y_{1}^{-}}\right|=\tau+1, \\
\widetilde{\tau}_{0} & =\left[\tau+1-\left(n_{1}+n_{2}+1\right)\right](\bmod (1+\theta))=\tau_{0} .
\end{aligned}
$$

Теперь заметим, что любую из пар гетероклинических точек траекторий $\Gamma_{12}$ и $\Gamma_{21}$ можно получить из исходной путем конечного числа элементарных переходов к такой паре, в которой одна из точек заменяется на ее итерацию отображения $f$. Поскольку, как мы показали, при каждом таком элементарном переходе к очередной паре точек величина $\tau_{0}$ не меняется, то она будет одной и той же и для любой пары гетероклинических точек. Лемма доказана.

Из доказательства леммы 3 нетрудно вывести по индукции формулу, позволяющую найти величину $\tau$ для произвольно выбранных пар гетероклинических точек на $U_{1}$ и $U_{2}$. Именно, рассмотрим следующие гетероклинические точки $T_{01}^{s_{1}} M_{1}^{+}$, $T_{01}^{-s_{2}} M_{1}^{-}, T_{02}^{s_{3}} M_{2}^{+}$и $T_{02}^{-s_{4}} M_{2}^{-}$, где $s_{1}, s_{2}, s_{3}$ и $s_{4}$-некоторые целые числа. Тогда новое значение $\tau$, которое обозначим как $\widetilde{\tau}$, будет связано с $\tau \equiv \tau\left(M_{1}^{+}, M_{1}^{-}, M_{2}^{+}, M_{2}^{-}\right)$ следуюшим образом:

$$
\widetilde{\tau}=\tau+\left(s_{1}+s_{2}\right)-\theta\left(s_{3}+s_{4}\right) .
$$

Теорема 6. Пусть $f, f^{\prime} \in H_{3}$ u $f$ u $f^{\prime}-\Omega$-сопряженьь, $\theta=\theta^{\prime}$ и $\theta$ иррационально. Тогда $\tau_{0}=\tau_{0}^{\prime}$.

ДокАЗАТЕльство. Пусть $M_{s}^{+}, M_{s}^{-}$и $M_{s}^{\prime}, M_{s}^{\prime}, s=1,2,-$ пары сопряженных гетероклинических точек. Покажем сначала, что величины $\tau$ и $\tau^{\prime}$, вычисленные по этим точкам, должны совпадать. Действительно, предположим, что $\tau<\tau^{\prime}$. Тогда рассмотрим следующие неравенства

$$
\begin{aligned}
& i<j \theta-\tau-S_{2}\left(\bar{k}_{1}, \bar{k}_{2}\right), \\
& i \geqslant j \theta-\tau^{\prime}+S_{2}^{\prime}\left(\bar{k}_{1}, \bar{k}_{2}\right) .
\end{aligned}
$$

Так как $\theta$ иррационально и $\tau<\tau^{\prime}$, то при достаточно больших $\bar{k}_{1}$ и $\bar{k}_{2}$ существует счетное множество натуральных чисел $i$ и $j$, удовлетворяющих первому неравенству в (4.8) и не удовлетворяющих второму. Как было разъяснено при доказательстве теоремы 5 , это означает, что у диффеоморфизма $f$ в любой окрестности контура 
$C$ найдутся седловые периодические траектории (например, однообходные), имеющие такие кодировки, каких не может быть у неблуждающих траекторий дифифеоморфизма $f^{\prime}$. Последнее в силу леммы 2 противоречит тому, что мы выбрали сопряженные пары гетероклинических точек. Аналогично рассматривается случай $\tau>\tau^{\prime}$, здесь достаточно поменять местами $f$ и $f^{\prime}$.

Теперь заметим, что равенство $\tau=\tau^{\prime}$ для сопряженных гетероклинических точек возможно лишь в случае, ког да $\tau_{0}=\tau_{0}^{\prime}$. Действительно, в силу коммутативности диаграммы из определения 2 должны выполняться соотношения

$$
\begin{aligned}
f^{m_{1}}\left(M_{1}^{-}\right) & =M_{2}^{+}, \quad f^{m_{2}}\left(M_{2}^{-}\right)=M_{1}^{+}, \\
f^{\prime} m_{1}\left(M_{1}^{\prime}\right) & =M_{2}^{\prime}, \quad f^{\prime} m_{2}\left(M_{2}^{\prime}\right)=M_{1}^{\prime}
\end{aligned}
$$

для некоторых целых $m_{1}$ и $m_{2}$. Из (4.6) и (4.9) получаем, что

$$
\begin{aligned}
\tau & =\tau_{0}+\left(m_{1}+m_{2}\right)+k(1+\theta) \\
\tau^{\prime} & =\tau_{0}^{\prime}+\left(m_{1}+m_{2}\right)+k^{\prime}(1+\theta)
\end{aligned}
$$

где $k$ и $k^{\prime}$ - некоторые целые числа. Но тогда

$$
\begin{aligned}
{\left[\tau-\tau^{\prime}\right](\bmod (1+\theta)) } & =\left[\tau_{0}-\tau_{0}^{\prime}+\left(k-k^{\prime}\right)(1+\theta)(\bmod (1+\theta))\right. \\
& =\left[\tau_{0}-\tau_{0}^{\prime}\right](\bmod (1+\theta))
\end{aligned}
$$

Таким образом, если $\tau_{0} \neq \tau_{0}^{\prime}$, то и $\tau \neq \tau^{\prime}$. Это завершает доказательство теоремы.

\section{§5. Достаточные условия $\Omega$-сопряженности при рациональном $\theta$}

Вопрос о достаточных условиях $\Omega$-сопряженности диффеоморфизмов третьего класса при иррациональном $\theta$ является весьма сложным и, вообще говоря, неразрешимым в полном объеме. Это связано с тем, что при иррациональном $\theta$ у таких диффеоморфизмов, помимо $\theta$ и $\tau_{0}$, сушествуют и другие модули $\Omega$-сопряженности и более того, как мы покажем ниже в $\S 5$, в $H_{3}$ будут плотны системы со счетным множеством модулей $\Omega$-сопряженности.

При рациональном $\theta$ ситуация совершенно другая. Здесь, аналогично случаю систем с негрубой гомоклинической траекторией [3], [4], можно показать, что почти все системы на $H_{3}$ допускают полное описание множества $N$ траекторий, целиком лежащих в некоторой достаточно малой окрестности $U$ гомоклинического контура. Правда, размеры этой окрестности будут зависеть от $\theta$. Именно, справедлива следующая

Tеорема 7. Пусть $f \in H_{3} u \theta=p / q$, где $p$ и $q$-взаимно простые натуральные числа. Пусть также $\tau_{0} q \notin \mathbb{Z}$. Тогда существуют такие $\bar{k}_{1}$ и $\bar{k}_{2}$, зависящие от $p, q$ и $\tau_{0}\left(\bar{k}_{1}, \bar{k}_{2} \rightarrow \infty\right.$, если $q \rightarrow \infty$ или $\left.\tau_{0} q \rightarrow k \in \mathbb{Z}\right)$, и специальная окрестность $U\left(\bar{k}_{1}, \bar{k}_{2}\right)$, что все траектории множества $N\left(\bar{k}_{1}, \bar{k}_{2}\right) \backslash \Gamma_{21}$ имеют седловой тип, и $\left.f\right|_{N}$ сопряжено с символической динамической системой $\widetilde{\Omega}$. 
ДокАЗАТЕЛЬСтво. В $N$ нет траекторий, которые пересекали бы последовательно полоски $\sigma_{j}^{12}$ и $\sigma_{i}^{01}$ такие, что $i$ и $j$ удовлетворяют неравенству (1.11), поскольку в этом случае $T_{21}\left(\sigma_{j}^{12}\right) \cap \sigma_{i}^{01}=\varnothing$. С другой стороны, траектории, пересекающие последовательно полоски $\sigma_{j}^{12}$ и $\sigma_{i}^{01}$ только для таких $i$ и $j$, для которых выполнено неравенство (1.10), будут седловыми (теоремы 1 и 4). Таким образом, если в $N$ есть траектория неседлового типа, то она имеет хотя бы две последовательные точки пересечения с полосками $\sigma_{j}^{12}$ и $\sigma_{i}^{01}$, номера $i$ и $j$ которых удовлетворяют неравенству

$$
\left|\gamma_{1}^{-i} y_{1}^{-}-c_{21} \lambda_{2}^{j} x_{2}^{+}\right| \leqslant\left|S_{i j}\left(\bar{k}_{1}, \bar{k}_{2}\right) / d_{21}\right| .
$$

Очевидно, это неравенство может иметь решения только лишь тогда, когда числа $\gamma_{1}^{-i}$ и $c_{21} \lambda_{2}^{j}$ одного знака (координаты на $U_{1}$ и $U_{2}$ введены так, что $y_{1}^{-}>0, x_{2}^{+}>0$ ). В этом случае неравенство (5.1), очевидно, можно переписать в виде

$$
|i-j \theta+\tau| \leqslant S_{3}\left(\bar{k}_{1}, \bar{k}_{2}\right)
$$

где $S_{3}\left(\bar{k}_{1}, \bar{k}_{2}\right) \rightarrow 0$ при $\bar{k}_{1}, \bar{k}_{2} \rightarrow \infty$.

Если бы $\theta$ было иррациональным, то неравенство (5.2) при любом $\tau$ и любых $\bar{k}_{1}$ и $\bar{k}_{2}$ имело бы счетное множество целочисленных решений. Но при $\theta$ рациональном, $\theta=p / q$, и при $\tau q \notin \mathbb{Z}$ неравенство (5.2) при достаточно больших $\bar{k}_{1}$ и $\bar{k}_{2}$, зависящих, вообше говоря, от $\theta$ и от того, насколько число $\tau q$ близко к целому, вообше не будет иметь целочисленных решений. Эта ситуация как раз и имеет место при выполнении условий теоремы. Действительно, число $\tau q$ будет целым тогда и только тогда, когда является целым число $\tau_{0} q$. Это вытекает из того, что в силу (4.6) $\tau$ и $\tau_{0}$ связаны соотношением

$$
\tau q=\left(n_{1}+n_{2}\right) q+m_{0}(q+p)+\tau_{0} q,
$$

где $m_{0}$ - некоторое целое число.

Итак, в предположении теоремы 7 неравенство (5.2), а вместе с ним и (5.1), при достаточно больших $\bar{k}_{1}$ и $\bar{k}_{2}$ не имеет целочисленных решений. Геометрический смысл этого факта состоит в том, что вершины подков $T_{21}\left(\sigma_{j}^{12}\right), j=\bar{k}_{2}, \bar{k}_{2}+1, \ldots$, попадают в промежутки между полосками $\sigma_{i}^{01}$ и $\sigma_{i+1}^{01}, i=\bar{k}_{1}, \bar{k}_{1}+1, \ldots$, и таким образом организуются только правильные и пустые пересечения. В этом случае $N\left(\bar{k}_{1}, \bar{k}_{2}\right)$ совпадает с $\widetilde{N}\left(\bar{k}_{1}, \bar{k}_{2}\right)$, и в силу теоремы $\left.4 f\right|_{N}$ сопряжено с $\widetilde{\Omega}$. Теорема доказана.

Рассмотрим теперь некоторые достаточные условия $\Omega$-сопряженности двух диффеоморфизмов третьего класса, значение инварианта $\theta$ у которых одинаково и рационально. При этом мы ограничимся случаем, ког да мультипликаторы точек $O_{1}$, $O_{2}$ и $O_{1}^{\prime}, O_{2}^{\prime}$ - положительны, ${ }^{3}$ т.е. будем рассматривать условия $\Omega$-сопряженности диффеоморфизмов, представленных на рис. 4в) или 4г), соответственно. Здесь имеет место следующая

\footnotetext{
${ }^{3}$ Случаи, когда мультипликаторы $\lambda_{1}$ или $\gamma_{2}$ отрицательны, рассматриваются, в принципе, аналогично, но также и включают некоторые специфические исследования свойств целочисленных решений неравенств (1.10)-(1.12), см., например, [4].
} 
Tеорема 8. Пусть $f, f^{\prime} \in H_{3} u \theta=\theta^{\prime}=p / q$. Предположим, что для некоторого челого $s$ точки $\tau_{0} q$ и $\tau_{0}^{\prime} q$ лежат в интервале $(s, s+1)$. Тогда существуют такие специальные окрестности $V$ и $V^{\prime}$ гомоклинических контуров $C$ и $C^{\prime}$, ито $\left.f\right|_{N(f)}$ сопряжсено с $\left.f^{\prime}\right|_{N\left(f^{\prime}\right)}$.

ДокаЗАтЕЛЬство. Рассмотрим, для определенности, опять случай, когда $f$ и $f^{\prime}$ - диффеоморфизмы такого же типа, как на рис. 4в), т.е. будем считать, что $\lambda_{s}>0, \gamma_{s}>0, c_{21}>0, d_{21}>0$. Для диффеоморфизма $f$ выберем гетероклинические точки $M_{1}^{-}, M_{2}^{+}$траектории $\Gamma_{12}$ и $M_{2}^{-}, M_{1}^{+}$траектории $\Gamma_{12}$. Пусть $m_{1}$ и $m_{2}$ такие целые числа, что $f^{m_{1}}\left(M_{1}^{-}\right)=M_{2}^{+}, f^{m_{2}}\left(M_{2}^{-}\right)=M_{1}^{+}$. Кроме того, в силу (4.6) сушествует такое целое $k$, что

$$
\tau=\tau_{0}+\left(m_{1}+m_{2}\right)+k(1+\theta) .
$$

Так как $\theta=p / q$ и $\tau_{0}=(s+\alpha) / q$, где $0<\alpha<1$, то неравенство (5.4) можно переписать в виде

$$
i-j \frac{p}{q}+\left(m_{1}+m_{2}+k\right)+\frac{s+k p}{q}+\frac{\alpha}{q}<S_{3}\left(\bar{k}_{1}, \bar{k}_{2}\right) .
$$

Очевидно, существуют такие достаточно большие $\bar{k}_{1}$ и $\bar{k}_{2}$, что неравенство (5.4) эквивалентно такому

$$
i-j \frac{p}{q}+\left(m_{1}+m_{2}+k\right)+\frac{s+k p}{q}+\frac{1}{2 q}<0 .
$$

у диффеоморфизма $f^{\prime}$ возьмем какие-либо гетероклинические точки $\widetilde{M}_{1}^{-}, \widetilde{M}_{2}^{+}$ траектории $\Gamma_{12}^{\prime}$ и $\widetilde{M}_{2}^{-}, \widetilde{M}_{1}^{+}$траектории $\Gamma_{12}^{\prime}$, но так, чтобы для тех же самых целых чисел $m_{1}$ и $m_{2}$, что и для $f$, выполнялись бы соотношения: $f^{\prime m_{1}}\left(\widetilde{M}_{1}^{-}\right)=\widetilde{M}_{2}^{+}$, $f^{\prime m_{2}}\left(\widetilde{M}_{2}^{-}\right)=\widetilde{M}_{1}^{+}$. В силу (4.6) сушествует такое целое $k^{\prime}$, что

$$
\widetilde{\tau}^{\prime}=\tau_{0}^{\prime}+\left(m_{1}+m_{2}\right)+k^{\prime}(1+\theta)
$$

где $\widetilde{\tau}^{\prime}$ - значение величины $\tau^{\prime}$, вычисленной по выбранным гетероклиническим точкам диффеоморфизма $f^{\prime}$.

Рассмотрим следующие гетероклинические точки:

$$
\begin{aligned}
& M_{1}^{\prime-} \equiv T_{01}^{\prime\left(k-k^{\prime}\right)}\left(\widetilde{M}_{1}^{-}\right), \quad M_{2}^{\prime+} \equiv T_{01}^{\prime\left(k-k^{\prime}\right)}\left(\widetilde{M}_{2}^{+}\right), \\
& M_{2}^{\prime-} \equiv \widetilde{M}_{2}^{-}, \quad M_{1}^{\prime}{ }^{+} \equiv \widetilde{M}_{1}^{+} .
\end{aligned}
$$

Из формулы (4.7) (в которой нужно положить $s_{1}=0, s_{2}=-\left(k-k^{\prime}\right), s_{3}=k-k^{\prime}$, $\left.s_{4}=0\right)$ получаем, что для величины $\tau^{\prime}$, вычисленной по точкам (5.7), имеет место следующее выражение

$$
\tau^{\prime}=\widetilde{\tau}^{\prime}-\left(k-k^{\prime}\right)-\theta\left(k-k^{\prime}\right)=\widetilde{\tau}^{\prime}-\left(k-k^{\prime}\right)(1+\theta) .
$$

В силу (5.6) тогда получаем, что

$$
\tau^{\prime}=\tau_{0}^{\prime}+\left(m_{1}+m_{2}\right)+k(1+\theta) .
$$


Так как $\theta=p / q$ и $\tau_{0}^{\prime}=\left(s+\alpha^{\prime}\right) / q$, где $0<\alpha^{\prime}<1$, то неравенство (1.10) для диффеоморфизма $f^{\prime}$ можно переписать в виде

$$
i-j \frac{p}{q}+\left(m_{1}+m_{2}+k\right)+\frac{s+k p}{q}+\frac{\alpha^{\prime}}{q}<S_{3}^{\prime}\left(\bar{k}_{1}{ }_{1}, \overline{k^{\prime}} 2\right) .
$$

Очевидно, сушествуют такие $\bar{k}_{1}^{\prime}$ и $\bar{k}_{2}^{\prime}$, что неравенство (5.9) будет эквивалентно неравенству (5.5).

Возьмем теперь $\widetilde{k}_{1}=\max \left\{\bar{k}_{1}, \bar{k}_{1}^{\prime}\right\}, \widetilde{k}_{2}=\max \left\{\bar{k}_{2}, \bar{k}_{2}^{\prime}\right\}$. Тогда из наших рассмотрений вытекает, что в специальных окрестностях $U \equiv U\left(\widetilde{k}_{1}, \widetilde{k}_{2}\right)$ и $U^{\prime} \equiv U^{\prime}\left(\widetilde{k}_{1}, \widetilde{k}_{2}\right)$ системы $\left.f\right|_{N}$ и $\left.f^{\prime}\right|_{N^{\prime}}$ будут сопряжены с системой $\widetilde{\Omega}$, удовлетворяюшей условиям 2.1)-2.4) (см. $\S 2)$, где $\bar{k}_{1}=\widetilde{k}_{1}, \bar{k}_{2}=\widetilde{k}_{1}$, а в условии 2.4) неравенство (1.10) нужно заменить на эквивалентное ему в данном случае неравенство (5.5). Теорема доказана.

\section{§6. Существование счетного множества $\Omega$-модулей}

В этом параграфе мы распространим результаты работ [5], [6], в которых установлено сушествование счетного множества $\Omega$-модулей у систем с негрубой гомоклинической траекторией, на случай систем с негрубым гетероклиническим контуром. Именно, будет доказана следующая

Теорема 9. В Н $_{3}$ плотны системь, имеющие счетное множество модулей $\Omega$-сопряженности.

ДоКАЗАТЕЛЬСТво. В основе доказательства этой теоремы лежит следуюшая лемма

ЛЕмма 4. В $H_{3}$ плотно множество $B$ такое, что любой диффеоморфизм из $B$ имеет грубую седловую периодическую траекторию с негрубой гомоклинической орбитой.

Эту лемму мы докажем ниже, а сейчас предположим, что она верна. Воспользуемся результатом работы [6], в которой показано, что любую систему с негрубой гомоклинической точкой можно сколь угодно мало возмутить (возмущением класса $C^{r-1}$ ) так, чтобы получить систему со счетным множеством грубых седловых периодических траекторий, каждая из которых имеет негрубую гомоклиническую орбиту. Заметим, что эти возмущения будут локализованы в малой окрестности негрубой гомоклинической траектории и, следовательно, не выводят из “пленки" $H_{3}$ диффеоморфизмов с негрубым гетероклиническим контуром третьего класса.

Рассмотрим теперь диффеоморфизм $f^{*}$, лежащий в $H_{3}$ и имеющий счетное число грубых седловых периодических траекторий $O_{1}^{*}, \ldots, O_{k}^{*}, \ldots$, каждая из которых имеет негрубую гомоклиническую орбиту, соответственно, $\Gamma_{k}^{*}$. Очевидно, $f^{*}$ лежит в пересечении счетного множества гладких банаховых многообразий $\mathscr{M}_{n}$ таких, что любой диффеоморфизм из $\mathscr{M}_{n}$ имеет $n$ близких к $O_{1}^{*}, \ldots, O_{n}^{*}$ периодических траекторий $\widetilde{O}_{1}^{*}, \ldots, \widetilde{O}_{n}^{*}$ с негрубыми гомоклиническими траекториями $\widetilde{\Gamma}_{1}^{*}, \ldots, \widetilde{\Gamma}_{n}^{*}$. Пусть $\nu_{k}, \rho_{k}\left(\left|\nu_{k}\right|<1,\left|\rho_{k}\right|>1\right)$ - мультипликаторы траектории $O_{k}^{*}$. 
В [3], [4] показано, что величины $\theta_{k}=-\ln \left|\nu_{k}\right| / \ln \left|\rho_{k}\right|$ являются модулями $\Omega$-сопряженности на $\mathscr{M}_{n}$. Очевидно, величины $\theta_{k}, k=1,2, \ldots$, являются независимыми, так как при разных $k$ выражаются через мультипликаторы разных периодических траекторий. Тогда по определению 1 диффеоморфизм $f^{*}$ имеет счетное множество $\Omega$-модулей. Это завершает доказательство теоремы.

Теперь осталось только доказать лемму 4. Рассмотрим однопараметрическое семейство $f_{\theta}$ диффеоморфизмов на $H_{3}$ и покажем, что для любого $\theta=\theta_{0}$ на интервале $\left(\theta_{0}-\varepsilon, \theta_{0}+\varepsilon\right)$ для любого $\varepsilon>0$ плотны значения $\theta^{*}$ такие, что диффеоморфизм $f_{\theta^{*}}$ имеет негрубую гомоклиническую орбиту точки $O_{1}$.

Зафиксируем $\theta=\theta_{0}$ и для диффеоморфизма $f_{\theta_{0}}$ рассмотрим множество пар $(i, j)$, для которых вьполнено неравенство (1.10). В случае $\lambda_{1}>0, \gamma_{2}>0, c_{21}>0$, $d_{21}>0$ оно принимает вид

$$
i<j \theta_{0}-\tau+S_{2}\left(\bar{k}_{1}, \bar{k}_{2}\right)
$$

Положим теперь $\theta=\theta_{0}-\varepsilon$ и рассмотрим для диффеоморфизма $f_{\theta_{0}-\varepsilon}$ множество пар $(i, j)$, для которых выполнено неравенство (1.11), т.е.

$$
i>j\left(\theta_{0}-\tau-\varepsilon\right)-S_{2}\left(\bar{k}_{1}, \bar{k}_{2}\right)
$$

Рассмотрим теперь множество пар $(i, j)$, для которых выполнены одновременно неравенства (6.1) и (6.2). Множество таких пар, очевидно, счетно для любого $\varepsilon>0$. Пусть $\left(i^{*}, j^{*}\right)$ - одна из таких пар. Для диффеоморфизма $f_{\theta_{0}}$ полоска $\sigma_{i^{*}}^{01}$ и подкова $T_{21}\left(\sigma_{j^{*}}^{21}\right)$ пересекаются правильно. Тогда будут пересекаться кривая

$$
W_{i^{*}}^{s} \equiv T_{01}^{-i^{*}}\left(T_{12}^{-1} W_{\mathrm{loc}}^{s}\left(O_{1}\right)\right) \subset W^{s}\left(O_{1}\right)
$$

лежашая в полоске $\sigma_{i^{*}}^{01}$, и кривые

$$
W_{i^{*} j^{*}}^{u} \equiv T_{21} T_{02}^{j^{*}} T_{12} T_{01}^{i^{*}} T_{21}\left(W_{\mathrm{loc}}^{u}\left(O_{1}\right)\right) \subset W^{u}\left(O_{1}\right),
$$

лежащие в подкове $T_{21}\left(\sigma_{j^{*}}^{21}\right)$. Точки пересечения этих кривых - это гомоклинические точки седла $O_{1}$. При $\theta=\theta_{0}-\varepsilon$ подкова $T_{21}\left(\sigma_{j^{*}}^{21}\right)$ и полоска $\sigma_{i^{*}}^{01}$ не пересекаются, поэтому нет и соответствующих гомоклинических точек. Следовательно, найдется такое $\theta^{*} \in\left(\theta_{0}-\varepsilon, \theta_{0}\right)$, при котором $f_{\theta^{*}}$ будет иметь негрубую гомоклиническую траекторию точки $O_{1}$. В случае диффеоморфизма, представленного на рис. 4 г), аналогично доказьвается плотность значений параметра $\theta$, при которых $f_{\theta}$ имеет негрубую гомоклиническую траекторию точки $O_{2}$.

Автор благодарит Л. П. Шильникова и Д. В. Тураева за полезные обсуждения. 


\section{Список литературы}

1. Palis J. A differentiable invariant of topological conjugacies and moduli of stability // Asterisque. 1978. V. 51. P. 335-346.

2. Гонченко C. В. Модули систем с негрубыми гомоклиническими траекториями (случаи диффеоморфизмов и векторных полей) // Методы качественной теории и теории бифуркаций. Межвуз. тематич. сб. науч. тр. Горький: Горьк. гос. ун-т, 1989. С. 34-49.

3. Гонченко С. В., Шильников Л. П. Инварианты $\Omega$-сопряженности диффеоморфизмов с негрубой гомоклинической траекторией // Укр. матем. журн. 1990. Т. 42 . № 2. C. $153-159$.

4. Гонченко С. В., Шильников Л. П. О модулях систем с негрубой гомоклинической кривой Пуанкаре // Изв. РАН. Сер. матем. 1992. Т. 56. №6. С. 1165-1197.

5. Гонченко С. В., Тураев Д. В., Шильников Л. П. О моделях с негрубой гомоклинической кривой Пуанкаре // ДАН СССР. 1991. Т. 320. № 2. С. 269-272.

6. Gonchenko S. V., Shil'nikov L.P., Turaev D. V. On models with non-rough Poincare homoclinic curves // Phys. D. 1993. V. 62. № 1-4. P. 1-14.

7. Гаврилов Н.К., Шильников Л. П. О трехмерных динамических системах, близких к системе с негрубой гомоклинической кривой, I), II) // Матем. сб. 1972. Т. 88. № 4 . C. $475-492 ; 1973$. Т. 90 . №1. C. 139-157.

8. Гонченко C. B. Нетривиальные гиперболические подмножества систем с негрубой гомоклинической кривой // Методы качественной теории дифференциальных уравнений. Межвуз. тематич. сб. науч. тр. Горький: Горьк. гос. ун-т, 1984. С. 89-102.

9. Гонченко С. В., Шильников Л. П. О динамических системах с негрубыми гомоклиническими кривьми // ДАН СССР. 1986. Т. 286. № 5. С. 1049-1053.

10. Шильников Л. П. Об одной задаче Пуанкаре-Биркгофа // Матем. сб. 1967. Т. 74 (116). № 4. C. $378-397$.

11. Гаврилов Н. К. О трехмерных динамических системах, имеющих негрубый гомоклинический контур // Матем. заметки. 1973. Т. 14. № 5. С. 687-696.

12. Шильников Л. П. Об одном случае существования счетного множества периодических дВижений // ДАН СССР. 1965. Т. 160. № 3. С. 558-561.

13. Арнольд В.И., Афраймович В. С., Ильяшенко Ю. С., Шильников Л. П. Теория бифуркаций // Итоги науки и техн. Совр. пробл. матем. Фундам. направления. Т. 5. М.: ВИНИТИ, 1986. С. 5-218.

14. Togawa Y. A modulus of 3-dimensional vector fields // Dynamical System Ergodic Theory. 1987. V. 7. P. 295-303.

15. Шильников Л. П. К вопросу о структуре расширенной окрестности грубого состояния равновесия типа седло-фокус // Матем. сб. 1970. Т. 81 (123). № 1. С. 92-103.

16. Овсянников И.М., Шильников Л. П. О системах с гомоклинической кривой седло-фокуса // Матем. сб. 1986. Т. 130 (172). № 4. С. 552-570.

17. Овсянников И. М., Шильников Л. П. Системы с гомоклинической кривой многомерного седло-фокуса и спиральный хаос // Матем. сб. 1991. Т. 140 (182). № 7. С. 1043-1073.

18. Гонченко С.В. К вопросу об условиях сопряженности двумерных диффеоморфизмов с негрубой гомоклинической траекторией // Методы качественной теории и теории бифуркаций. Межвуз. тематич. сб. науч. тр. Горький: Горьк. гос. ун-т, 1990. С. 5-19.

Научно исследовательский институт

Поступила в редакцию прикладной математики и кибернетики при Нижегородском государственном университете (НИИ ПМК)

11.01 .1996

E-mail: gonchenko@focus.nnov.su 